\title{
Effectively Optimizing Production of Horizontal Wells in Homogeneous Oil Reservoirs
}

\author{
Hector Ngozi Akangbou*, Martin Burby, Ghasem Nasr \\ Petroleum Technology and Spray Research Group, School of Computing Science and \\ Engineering, University of Salford, Manchester, United Kingdom, M5 4WT.
}

\section{Correspondance email: hectorakangbou@gmail.com}

\begin{abstract}
Horizontal well applications have been predominant since their conception, for reasons such as effective depletion of oil reservoirs and especially in water cresting, gas cresting or water and gas cresting applications due to the casings enhanced exposure to the reservoir. Cresting is hugely dependent on oil production rate, pressure drawdown and can negatively influence the degree of depletion as well as the overall recovery efficiency of oil reservoirs. This paper presents a novel procedure of mimicking horizontal wells aimed at investigating experimentally the effect of varying inclined sections (having different vertical and horizontal displacements) of horizontal wells at low angles of inclination $\left(15^{\circ}-30^{\circ}\right)$ in a homogeneous reservoir underlain by a strong bottom aquifer and overlain by a considerable gas cap drive occurring simultaneously.

The results for the performance of the different horizontal well geometries in terms of cumulative oil recovery and Water-Oil-Ratio; over a fixed liquid production time were compared. From the results obtained, it was observed that the short radius well with $30^{\circ}$ angle of inclination and ratio of vertical displacement of the inclined section to reservoir height of 0.07 resulted in the highest oil recovery of 38.73\%. Using the presented procedure, 5.60\% increment in oil was recovered with $11.40 \%$ reduction in cumulative produced water were observed between the best and worst cases from the same reservoir. At higher withdrawal rates and pressure drop, long radii wells are recommended due to cresting delay ability while improving oil recovery.
\end{abstract}

\section{Keywords}

Cresting, optimization, oil recovery, inclined section, Inclination angle, homogeneity

\section{Introduction}

Drilling of horizontal wells have been common practice (Permadi, 1996) for almost a century. The reason for this is their ability to access difficult to target oil pay zones and high productivity, by effectively delaying water and gas breakthrough times (Al Zarafi, 1993, Chen, 1993, Coffin, 1993, Murphy, 1990, Sherrard et al., 1987). The productivity of horizontal wells depends to a large extent on the total length and distribution of the open intervals (Goode and Wilkinson, 1991). Horizontal wells are a preferred candidate to vertical wells due to more reservoir exposure of its laterals resulting in a lower pressure drop at the same withdrawal rate, which is important for minimizing cresting effect. At static condition, the reservoir gas, oil and water phases are separated by gravity, in order of their density 
differences (Balazs et al., 2009, Beveridge et al., 1970, Singhal, 1996). Moments after production starts, the original plane interfaces between bottom water and oil and the overlying gas and oil become distorted (Permadi and Jayadi, 2010) rapidly as a result of pressure drawdown being greater than the hydrostatic pressure that exist between the oil and gas (gas cresting), oil and water (water cresting) or water, oil and gas (water and gas cresting) occurring simultaneously.

Cresting in horizontal wells unlike coning in vertical wells is a well-known reservoir problem, often described as the protruded, crest-like movement of water and or gas in an oil reservoir towards the perforation of a horizontal well as a result of the imbalance of gravitational and viscous forces (Kromah and Dawe, 2008, Makinde et al., 2011, Permadi, 1996). The water and gas move up and down respectively towards the perforations of the well, while displacing the oil along its path and after the breakthrough time elapses, insurgence of water and or gas will be experienced. At this point, factors such as liquid production rate (Lozada et al., 2011) or withdrawal rate, ratio of water-to-oil mobility, ratio of vertical-to-horizontal permeability, distance from the wellbore to oil-water contact, productive length of the wellbore, and both the size and shape of drainage area will determine the water cut increment (Permadi and Jayadi, 2010, Yang and Wattenbarger, 1991). Water cresting is known to have an adverse effect in oil producing reservoirs (Weijun et al., 2014) and could lead to the early shutting-in of producing wells. Due to the increased volume of effluents (water and gas) produced over time, cresting is considered uneconomical because more money is spent in handling these effluents: the water is known to be corrosive in nature (Smith and Pirson, 1963) while the gas could damage surface facilities, both influencing the overall recovery efficiency of the oil reservoirs.

Most research on water and gas cresting have focused on critical rates, breakthrough times, its delay and prevention, but very few have considered the inclined section at low angles of inclination for optimization purposes for horizontal wells in oil reservoir faced with cresting problems. Benamara and Tiab, 2001, investigated the effect of withdrawal rate, perforated thickness for vertical wells, horizontal well length and reservoir parameters in the Gas-OilRatio (GOR) post breakthrough behavior. Balazs et al., 2009, investigated water and gas cresting in horizontal producing wells using an experimental model. The sole aim of their research was to provide fluid mechanics-based engineering guidelines for optimizing the productivity in horizontal wells. Makinde et al., 2011 numerically studied the performance of a horizontal well after breakthrough of water and further performed sensitivity analysis by setting a base case model and varying reservoir parameters (oil production rate, vertical and horizontal permeability, length of perforation, height above perforation, area extent of reservoir and the porosity of the formation) and production rate independently. Freeborn et al., 1990, undertook a case study of water cresting in South Jenner pool, a thin oil rim reservoir with thick bottom water. Medium and long radii wells of $420 \mathrm{~m}$ and $1042 \mathrm{~m}$ horizontal displacements respectively were drilled to determine their inflow performances compared to a vertical well in the presence of bottom water. When the production results were reviewed it was observed that the maximum production rate was highest for the long radius well however they indicated that the possible causes of poor performance of the short radius well was due to the cemented completion in the pay zone and the ineffectiveness of 
jet perforation in penetrating drilling damage. In addition, the water cut over time for each well was not reported. (Freeborn et al., 1990) numerically investigated different placement of the horizontal well from the top of the reservoir and observed that there was a decline in oil reserves produced when the well is closer to the OWC and water cresting is more likely to occur faster due to the upward water flood provided by the bottom water. A more comprehensive and general review on cresting can be found in (Makinde et al., 2011, Permadi and Jayadi, 2010).

Owing to the fact that cresting is a natural phenomenon which will occur at some point in the production life of a horizontal well despite producing at a water and gas-free oil rate 'critical rate' (Leemhuis et al., 2007), there is a need to determine an optimum well geometry for better oil recovery in reservoirs with cresting problems. Previous researchers have investigated the use of horizontal wells in reducing cresting effects but have only considered the effect of varying the lateral lengths and measured depths (MD) of inclined or deviated wells without considering the effect of the steepness of such wells on its performance such as in the works of Balazs et al., 2009. In this regard, a novel procedure for mimicking inclined or deviated wells was developed for the purpose of this research. This procedure involved varying the inclined section of the horizontal well without altering the lengths of the laterals (having same completion mechanism) and vertical (main bore) sections of the horizontal well unlike in the works of Freeborn et al., 1990. This was possible using compression and pneumatic fittings. Using these fittings, the modeled inclined sections can be fitted to the lateral and main bore sections with ease. This instigates a change in the measured depths, true vertical depth (TVD), horizontal and vertical displacements of horizontal wells, vertical and horizontal displacements of the inclined sections at any preferred angle of inclination.

\section{Experiment description and procedure}

The facility used in this investigation is illustrated in Figure 1. The water and gas-cresting rig consists of a reservoir, $0.45 \mathrm{~m}$ long, $0.10 \mathrm{~m}$ wide and $0.43 \mathrm{~m}$ high. The reservoir was made of clear acrylic [Poly (methyl methacrylate)] for visibility of the water and gas cresting process and had a free surface for easy filling of the polymer pellets (porous media). The water inlet points 1 and 2 were for the water feed to the reservoir, which was taken from a water storage tank. In this study, the horizontal wells were considered to have configurations as described in Table 1 and 2. The horizontal wells had an internal diameter of $0.006 \mathrm{~m}$ and external diameter, $0.008 \mathrm{~m}$ and a fixed lateral section of $0.305 \mathrm{~m}$, exposed to the reservoir. These wells were constructed and coupled to the reservoir. The constructed inclined sections of $15^{\circ}-30^{\circ}$ angles were fitted to the vertical and lateral sections of the horizontal well using compression fittings. Production was instigated and stopped using a ball valve. A comprehensive summary of the dimensions for the different geometries of the horizontal well models used in this investigation, such as the measured depth, true vertical depth, horizontal displacement $\left(H_{d}\right)$ of the inclined section as well as the ratio of the Vertical displacement of the inclined section to reservoir height $\left(V_{d} / H_{r}\right)$, are illustrated in Tables 1 and 2 . For the case of this investigation, Cases-1A, 1B and 2A were considered long radii, Cases-3A, $2 \mathrm{~B}$ and $1 \mathrm{C}$, medium radii wells, while Cases $3 \mathrm{C}$, $3 \mathrm{~B}$ and $2 \mathrm{C}$ were short radii wells. The radius of arc $r$, for each inclined section was calculated using equation 1. 


$$
l=\frac{n^{o}}{360^{o}} \times 2 \pi r
$$

Where $l$ is the length of arc in meters, $n^{o}$ is the angle of inclination in degrees, $r$ is the radius of arc in meters and $\pi=3.142$.

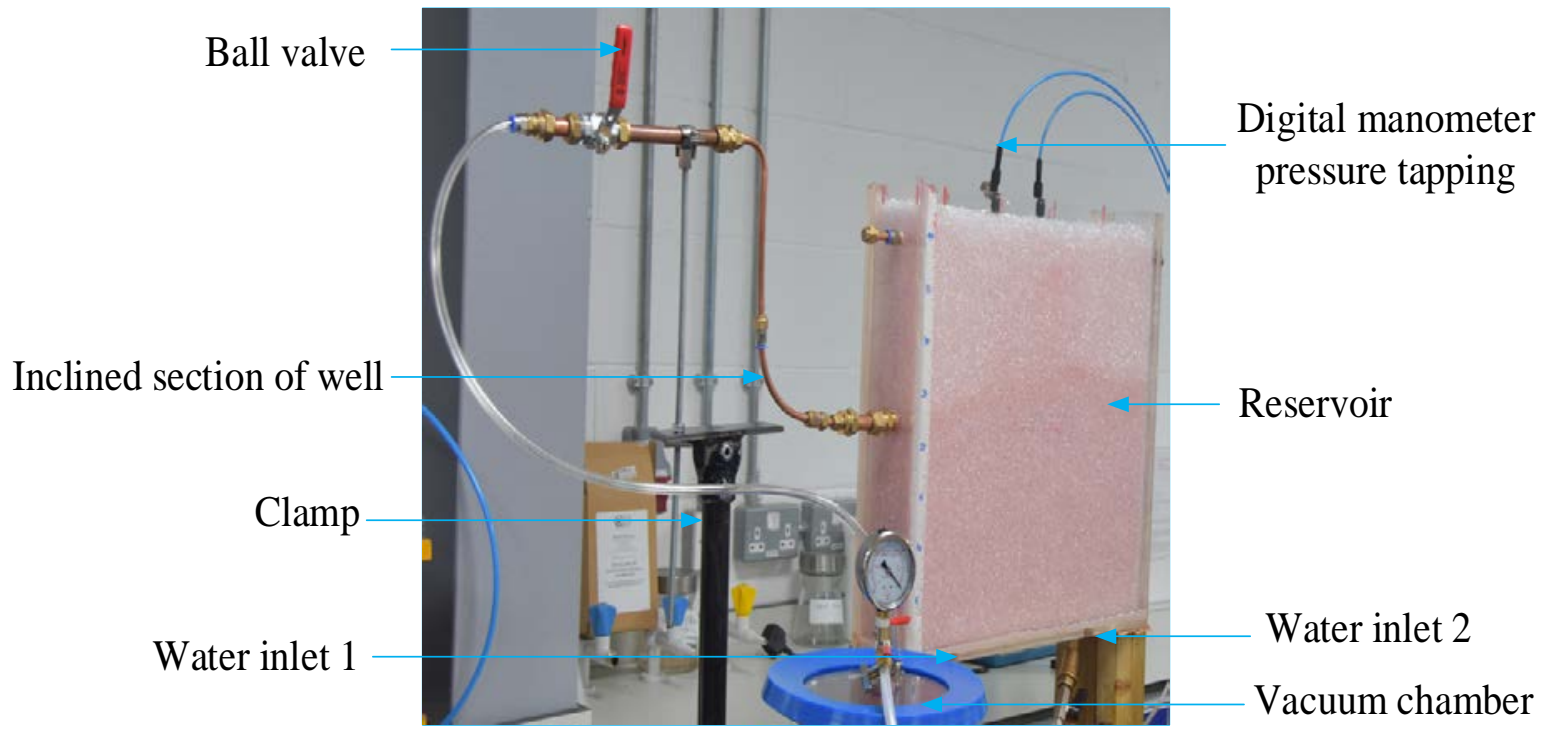

Figure1: Water and gas cresting facility.

The influx of fluid into the fixed perforated lateral section of the horizontal was radial in nature, having four shots per cross-section. The lateral distance between perforations was $0.05 \mathrm{~m}$, with hole-sizes of $0.002 \mathrm{~m}$. The reservoir type being modeled in this investigation was an oil reservoir; with the oil sandwiched between strong water bottom aquifer and a considerable gas cap drive. A strong bottom water drive was achieved with a total bottom water flow rate of $0.03 \mathrm{~kg} / \mathrm{s}$ through two inlet points located at the bottom of the reservoir to enable uniform water distribution. The bottom water was considered strong since the mass flow rate of the injected bottom water was greater than the overall approximate liquid mass flow rate $(0.01 \mathrm{~kg} / \mathrm{s})$ for all cases while the considerable gas cap drive was modeled at atmospheric pressure. Silicone oil [Poly (dimethylsiloxane)] was the oil used in this investigation due to its non-stain, non-sticky nature and most importantly it is insoluble in water at low and intermediate pressures.

Table 1: Geometry of horizontal wells [exp. $\left.{ }^{-1}(\mathrm{~m})\right]$

\begin{tabular}{|c|c|c|c|c|c|c|c|c|}
\hline \multirow[t]{2}{*}{ Cases } & \multirow{2}{*}{$\begin{array}{l}\text { Angle of } \\
\text { inclination } \\
\text { (Degrees) }\end{array}$} & \multirow[t]{2}{*}{ MD } & \multirow[t]{2}{*}{ TVD } & \multicolumn{2}{|c|}{$\begin{array}{l}\text { Build section } \\
\text { Measurement }\end{array}$} & \multirow[t]{2}{*}{$\mathrm{V}_{\mathrm{d}} / \mathrm{H}_{\mathrm{r}}$} & \multirow[t]{2}{*}{$\begin{array}{l}\text { Main } \\
\text { bore }\end{array}$} & \multirow{2}{*}{$\begin{array}{l}\text { Lateral } \\
\text { length } \\
\text { outside } \\
\text { reservoir }\end{array}$} \\
\hline & & & & $V_{d}$ & $\mathrm{H}_{\mathrm{d}}$ & & & \\
\hline Case-1A & 15 & 7.18 & 2.17 & 0.77 & 1.02 & 0.54 & 1.40 & 1.66 \\
\hline Case-1B & 23 & 7.01 & 2.03 & 0.63 & 0.93 & 0.43 & 1.40 & 1.66 \\
\hline
\end{tabular}




\begin{tabular}{lllllllll}
\hline Case-1C & 30 & 6.90 & 1.94 & 0.54 & 0.85 & 0.38 & 1.40 & 1.66 \\
Case-2A & 15 & 6.83 & 1.90 & 0.50 & 0.80 & 0.35 & 1.40 & 1.66 \\
Case-2B & 23 & 6.76 & 1.87 & 0.47 & 0.78 & 0.32 & 1.40 & 1.66 \\
Case-2C & 30 & 6.64 & 1.74 & 0.34 & 0.73 & 0.21 & 1.40 & 1.66 \\
Case-3A & 15 & 6.48 & 1.72 & 0.32 & 0.49 & 0.22 & 1.40 & 1.66 \\
Case-3B & 23 & 6.40 & 1.69 & 0.29 & 0.44 & 0.20 & 1.40 & 1.66 \\
Case-3C & 30 & 6.34 & 1.67 & 0.27 & 0.41 & 0.19 & 1.40 & 1.66 \\
\hline
\end{tabular}

Table 2: Geometry of horizontal wells contd. $\left[\exp .^{-1}(\mathrm{~m})\right]$

\begin{tabular}{ccccccc}
$\begin{array}{c}\text { Horizontal } \\
\text { departure of } \\
\text { well }\end{array}$ & $\begin{array}{c}\text { Length of } \\
\text { horizontal } \\
\text { lateral } \\
\text { section }\end{array}$ & $\begin{array}{c}\text { Distance } \\
\text { between } \\
\text { perforation }\end{array}$ & $\begin{array}{c}\text { Distance } \\
\text { from bridge } \\
\text { block to } \\
\text { first } \\
\text { perforation }\end{array}$ & $\begin{array}{c}\text { Length } \\
\text { of lateral } \\
\text { inside } \\
\text { reservoir }\end{array}$ & $\begin{array}{c}\text { Arc } \\
\text { Length }\end{array}$ & $\begin{array}{c}\text { Arc } \\
\text { radius }\end{array}$ \\
\hline 5.75 & 4.73 & 0.50 & 0.40 & 3.05 & 1.05 & 4.02 \\
\hline 5.66 & 4.73 & 0.50 & 0.40 & 3.05 & 0.88 & 2.23 \\
\hline 5.58 & 4.73 & 0.50 & 0.40 & 3.05 & 0.78 & 1.49 \\
\hline 5.53 & 4.73 & 0.50 & 0.40 & 3.05 & 0.70 & 2.68 \\
5.51 & 4.73 & 0.50 & 0.40 & 3.05 & 0.63 & 1.59 \\
\hline 5.46 & 4.73 & 0.50 & 0.40 & 3.05 & 0.51 & 0.98 \\
5.22 & 4.73 & 0.50 & 0.40 & 3.05 & 0.35 & 1.34 \\
5.17 & 4.73 & 0.50 & 0.40 & 3.05 & 0.27 & 0.70 \\
5.14 & 4.73 & 0.50 & 0.40 & 3.05 & 0.21 & 0.40 \\
\hline
\end{tabular}

For effective visualization of the water and gas cresting process, the water used was colored with fluorescein Sodium Dye-Fluorescent tracer, while clear-shiny plastic pellets were used as the porous media to distinguish the gas zone from the oil zone. Polymer pellets were first inserted to the required level ( $0.43 \mathrm{~m}$ from the base of the reservoir). Colored water was then pumped through the bottom inlets to the required WOC level $(0.03 \mathrm{~m}$ from the base of the reservoir). Silicone oil was introduced into the reservoir from the top of the reservoir to achieve a uniform WOC interface and until a GOC level of $0.37 \mathrm{~m}$ was attained prior to the start of production. The water was supplied at constant rate and pressure while producing oil. The production time for each scenario was 300 seconds (time enough for water and gas to breakthrough into the wellbore). Each analysis was run three times and the average values taken.

The cresting behavior of the water and gas was recorded using a video camera to enable the process to be analyzed frame by frame. The production mechanism of the silicone oil was by capillarity and pressure differential provided by a vacuum at a constant negative pressure of -4.351 Psig. However, for the purpose of sensitivity analysis, operating pressures of -7.25 and -10.15 Psig were used in a number of cases. The pressure variation in the reservoir was 
measured using a digital manometer with probes placed at fixed points $(0.18 \mathrm{~m}$ from top-left and right edges respectively) and depth of $0.22 \mathrm{~m}$ in the reservoir.

\subsection{Assumptions}

There were a number of assumptions that were considered with the experimental setup:

- The Water-Oil and Gas-Oil interfaces were assumed to be straight lines.

- Capillarity experienced at the different inclined sections were assumed to be negligible.

- $\quad$ The reservoir was assumed to have a free top surface.

\subsection{Total porosity and permeability determination}

Total porosity, usually represented in fraction or percentage is a measure of the pore volume to the total volume of a representative sample of the medium. The reservoir was filled with polymer pellets of diameters between $0.002 \mathrm{~m}$ and $0.003 \mathrm{~m}$ in size to instigate a homogeneous but irregular packed porous media. Although the reservoir does not mimic a typical homogeneous reservoir, it was considered homogeneous based on the same grain sizes and high-interconnected pore spaces visible in the free ray CT-Scanned images of the reservoir sample (Figure 2). This was possible by filling a cylindrical shaped vessel with the samesized pellets and performed a free ray scan using the GE Phoenix v|tome|x high-resolution CT-Scanner.

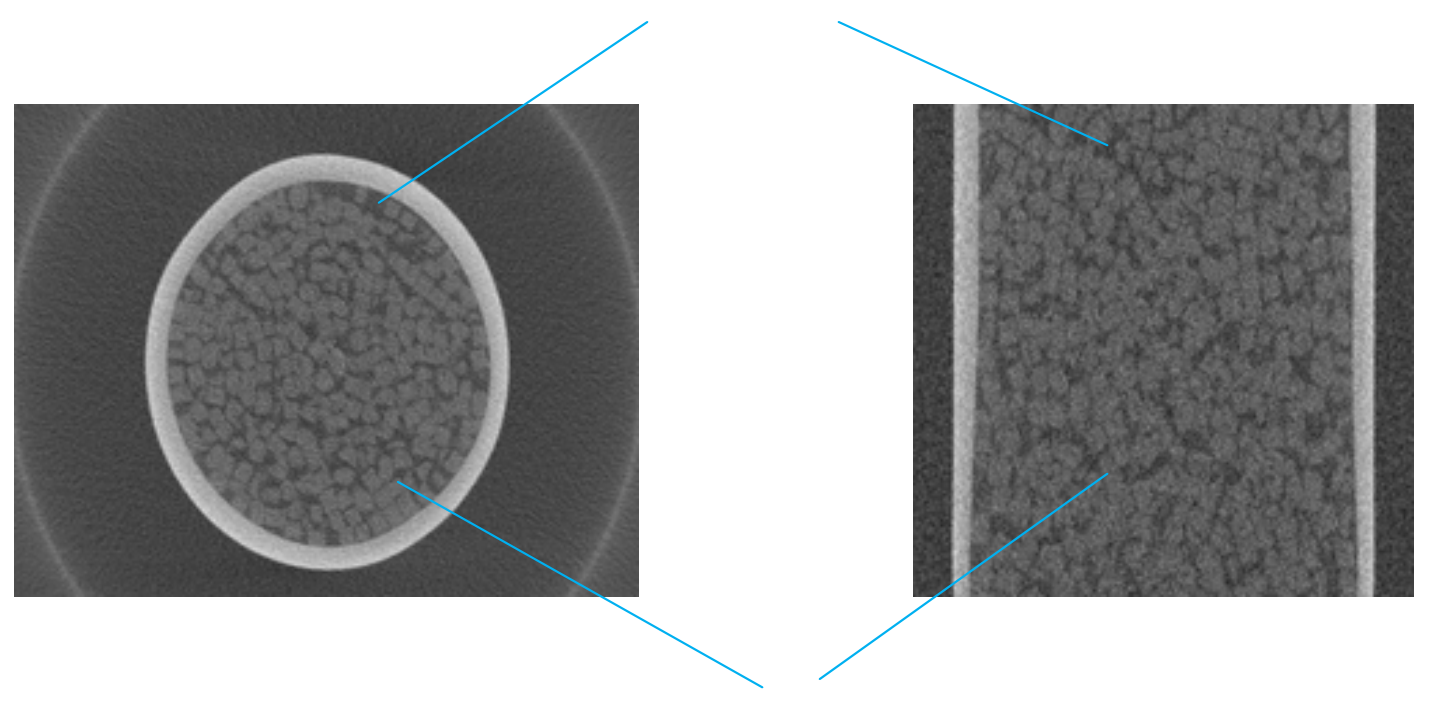

Figure 2: A free ray CT-Scan of reservoir sample showing pore spaces and grains.

The porous media used was polyethylene terephthalate (PET), with density $1302 \mathrm{~kg} / \mathrm{m}^{3}$. The total porosity of the reservoir was therefore high due to the small grain size of the porous media, achieving a calculated total porosity of $19.10 \%$, using equation 2 . 


$$
\emptyset=\frac{v-v_{s}}{v}=\frac{v_{p}}{v}
$$

Where $\varnothing$ is the total porosity in fraction, $v$ is the bulk volume of the reservoir in cubic meters, $v_{\mathrm{s}}$ is the total volume of the polymer pellet in cubic meter and $v_{\mathrm{p}}$ is the volume of the pore space in cubic meter.

Permeability is a property that significantly affects cresting and depends to a large extent on porosity of the given medium, such as the pore or grain size, packing of the grains, shape of the grains and the grain size distribution. The permeability of the oil, water and gas were determined from steady state experiments and using Darcy's linear equation (Equation 3). Figure 3 demonstrates how the permeability for gas (air) was determined. Compressed air was used as the source gas delivered at an upstream pressure (read from the in-line air regulator) to the top-inlet of the Fancher sample holder. The downstream pressure from the bottom-outlet of the sample holder was read from the pressure gauge at an airflow rate read from the airflow meter. Similar set up was used for oil and water permeability determination. The values of these permeabilities are in millidarcy and other reservoir parameters are summarized in Table 3.

$$
Q=\frac{k A\left(P_{2}-P_{1}\right)}{\mu L}
$$

Where $Q$ is the flow rate in cubic centimeter per second, $\mu$ is the viscosity of the fluid in centipoise, $k$ is the permeability in Darcy, $P_{2}$ is the Upstream Pressure in atmospheres, $P_{1}$ is the downstream pressure in atmospheres, $L$ is the length of flow in centimeters and $A$ is the cross-sectional area of flow in centimeters.

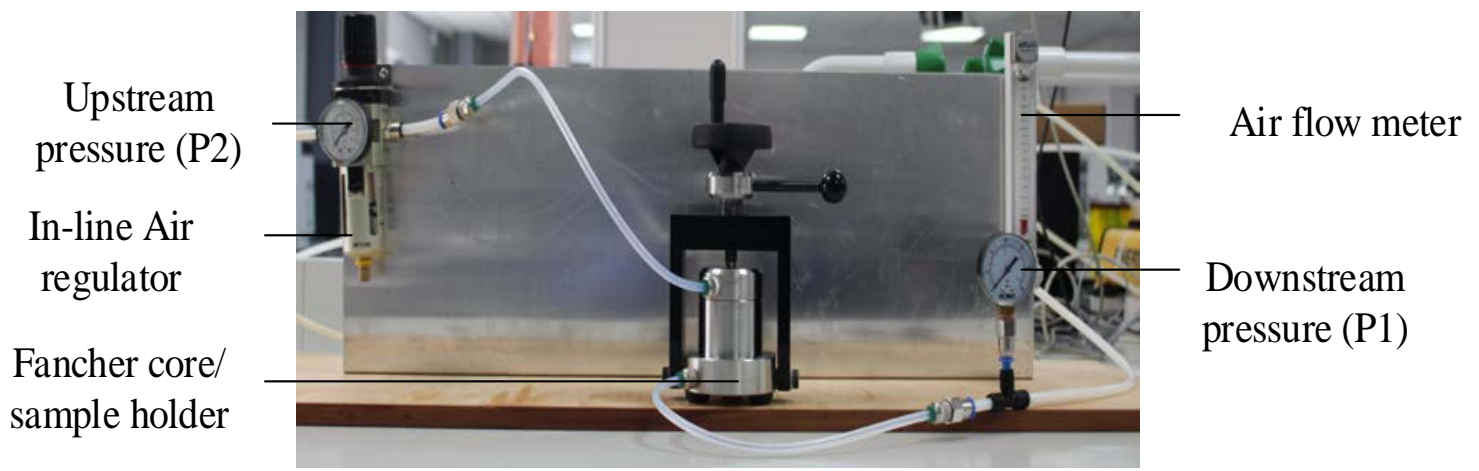

Figure 3: Set up for gas permeability determination

Table 3: Reservoir data.

\begin{tabular}{cc} 
Parameter & Values \\
\hline Viscosity of silicone oil (cP) & 50 \\
Viscosity of water (cP) & 1.004 \\
Permeability of silicone oil (md) $\left(\mathrm{exp}^{-2}\right.$ ) & 0.11 \\
Permeability of gas (md) (exp.- ${ }^{-2}$ & 0.44 \\
\hline
\end{tabular}




\begin{tabular}{cc} 
Permeability of water (md) $\left(\mathrm{exp}^{-2}\right)$ & 0.29 \\
Density of silicone oil $\left(\mathrm{Kg} / \mathrm{m}^{3}\right)$ & 972 \\
Density of dyed water $\left(\mathrm{Kg} / \mathrm{m}^{3}\right)$ & 998 \\
\hline
\end{tabular}

\subsection{Horizontal well placement and scaling}

The placement of a horizontal well is directly related to its performance, especially in oil reservoirs that experience cresting problems. Due to the nature of the reservoir considered in this study, the well was placed at a distance closer to the GOC such that both water and gas are produced at approximately the same time of 140 seconds. The horizontal lateral exposed to the reservoir was fixed at 0.67 times the length of the reservoir and 0.5 times the height of the reservoir. For scaling purposes, the arc lengths, vertical and horizontal displacements of the inclined section, as well as the ratio of vertical to reservoir height were plotted against measured depth of the horizontal wells, as illustrated in Figures 4-8 respectively. Figure 4, illustrates a plot of the horizontal well's lengths of arc against the measured depth. In this figure, the measured depth is seen to increase linearly with increase in length of arc. In Figure 5 , an increase in horizontal and vertical displacement of the inclined section results in increase in measured depth of the horizontal wells. The measured depth is seen to increase with increase in ratio of vertical displacement and reservoir height depicted in Figure 6. Figure 7 illustrates the plot of horizontal displacement of the inclined section versus Angle of inclination. As seen in this figure, the horizontal displacements of the inclined section decrease geometrically with increase in inclination angle. In Figure 8, the vertical displacement is seen to decrease with increasing angle of inclination. Figures 4-8 can be interpolated to match field conditions.

\section{Arc Length versus Measured Depth}

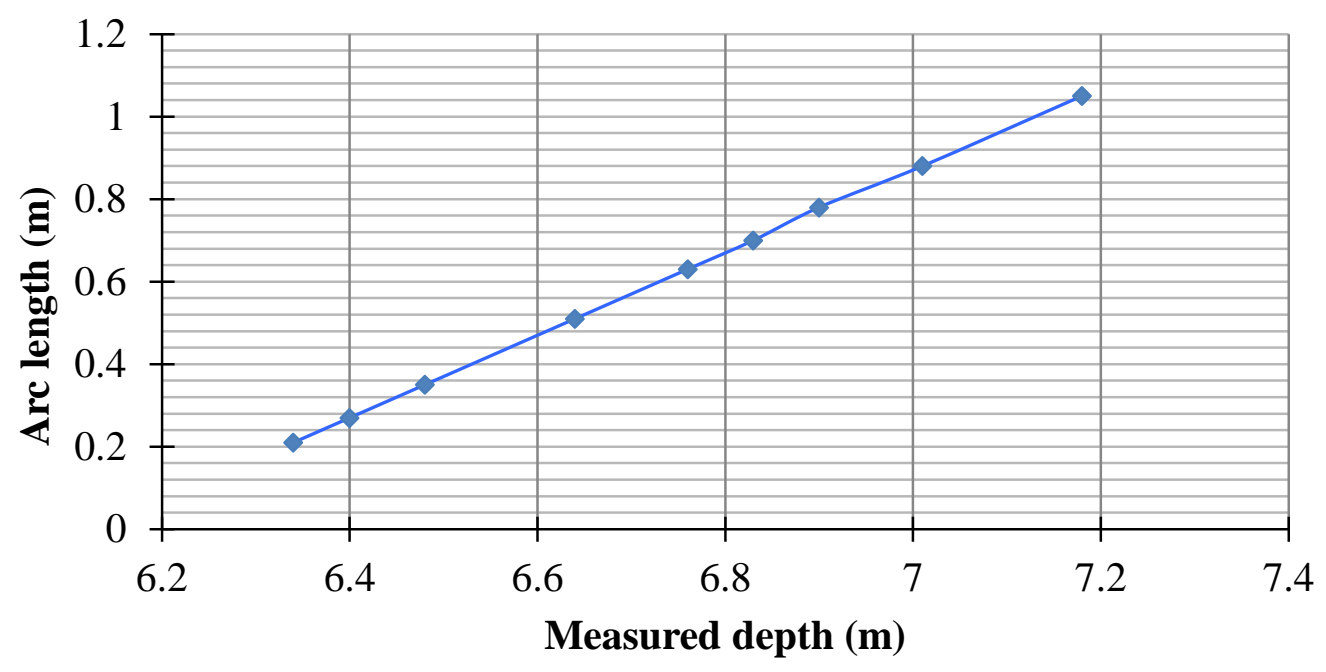

Figure 4: Plot of arc length versus measured depth. 


\section{Vertical and Horizontal Displacements of Inclined Section versus Measured Depth}

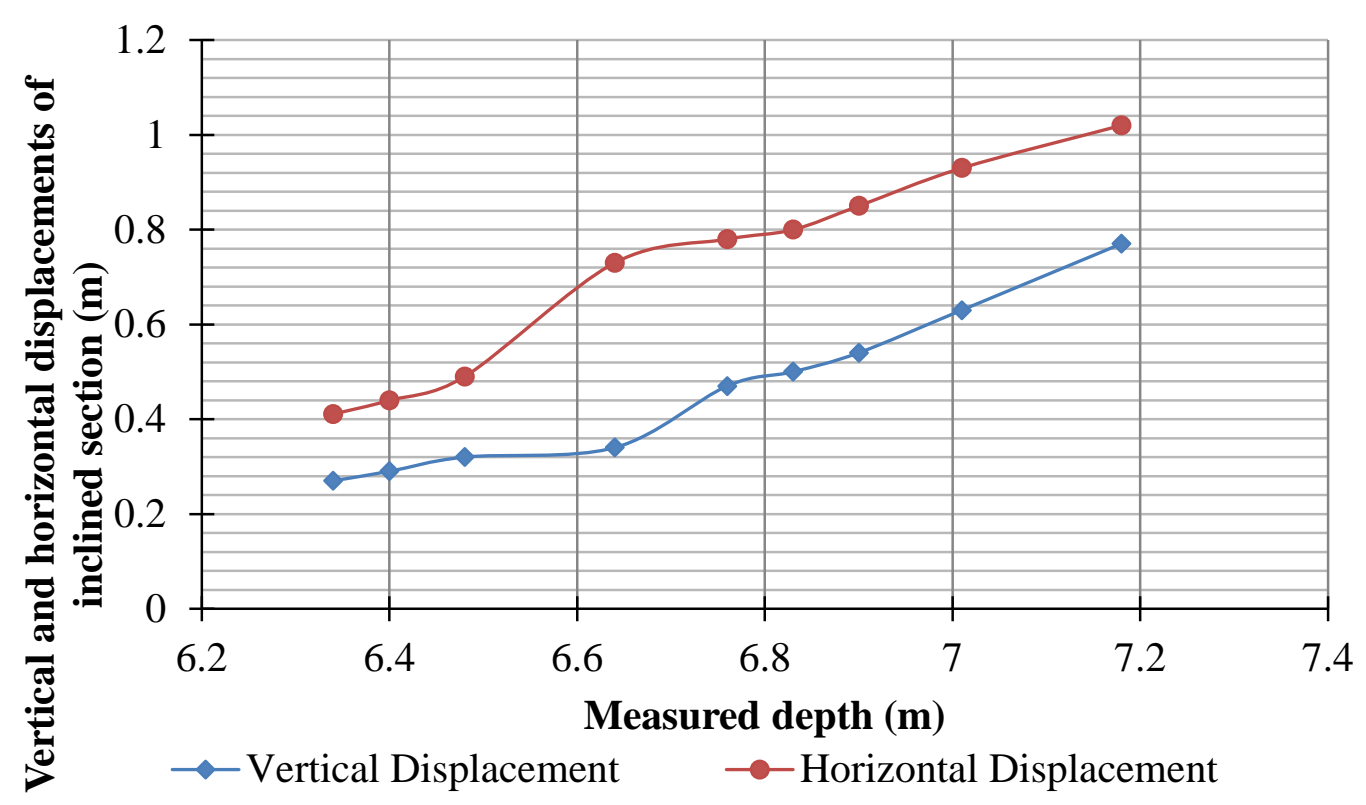

Figure 5: Plot of vertical and horizontal displacements of inclined section of wells against measured depth.

\section{Ratio of Vertical Displacement and Reservoir Height versus Measued Depth}

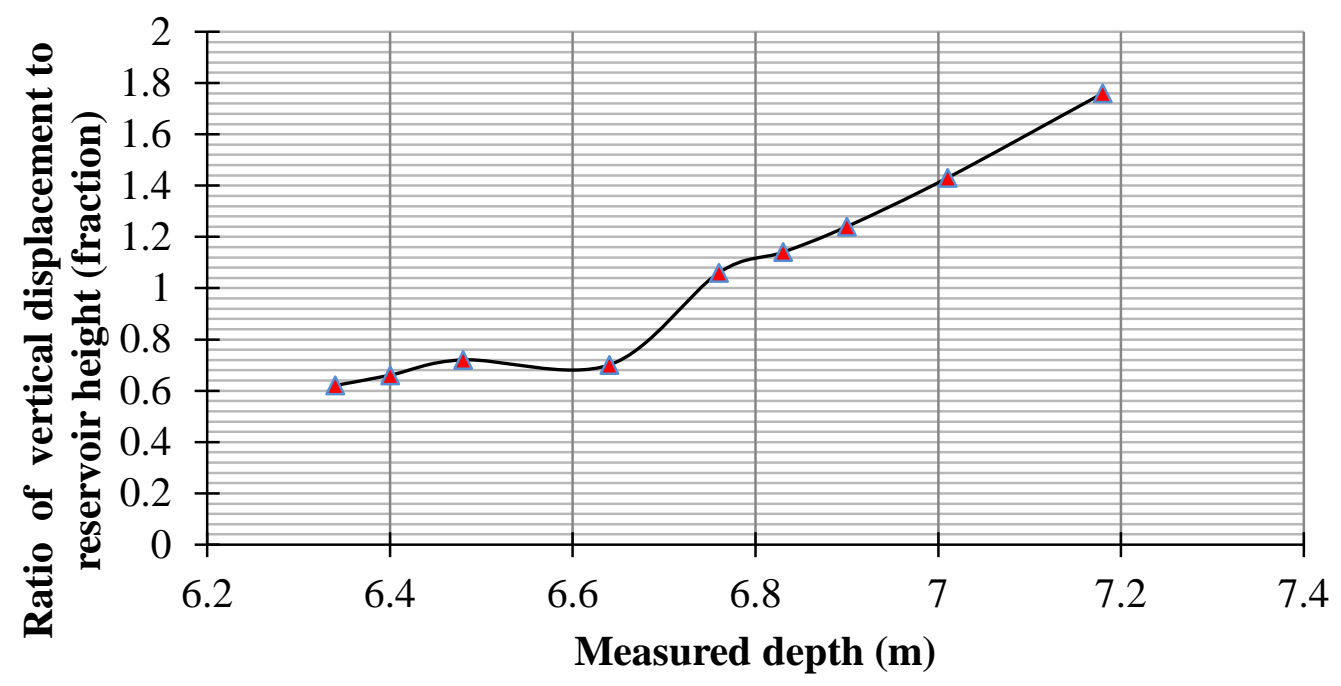

Figure 6: Plot of ratio of vertical displacement and reservoir height versus measured depth. 


\section{Horizontal Displacement of Inclined Section versus Angle of}

Inclination

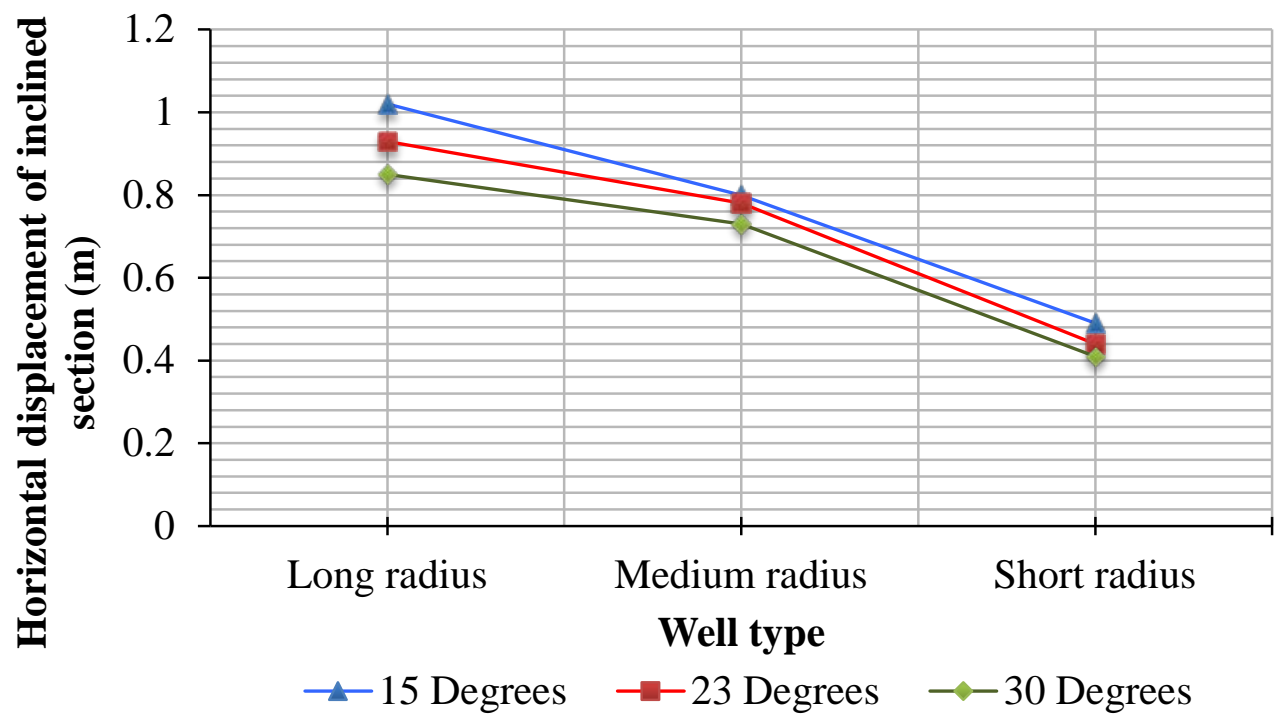

Figure 7:Plot of horizontal displacement versus angle of inclination.

Vertical Displacement of Inclined Section versus Angle of Inclination

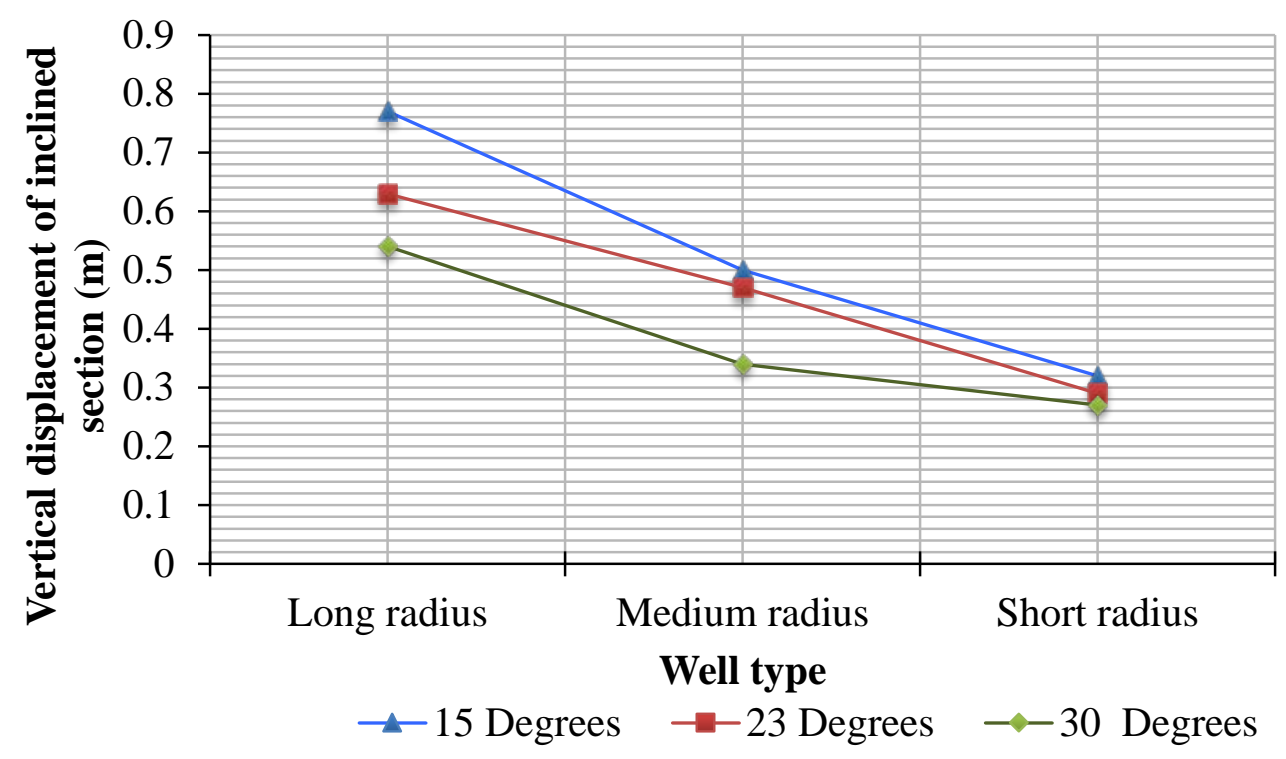

Figure 8: Plot of vertical displacement versus angle of inclination.

\subsection{Reynolds number}


Reynolds number (Re) is a dimensionless number usually applied to determine the fluid flow regime prediction, whether laminar partially turbulent or turbulent. This dimensionless number has been applied for the purpose of generalization of results from the scaled model used in this study. Since fluid flow in this study is through a pipe (horizontal well), equation 4 was used to estimate the Reynolds number. The Reynolds numbers for all horizontal well cases shown in Table 4 were less than $2000\left(R_{e}<2000\right)$ specifically $<1$, hence depicting laminar flow due to dominant viscous forces to forces of inertia. A correlation between the Reynolds number and measured depths of all horizontal well cases as well as the effect of change in oil production rate (change in outlet / surface pressure) on the Reynolds number for Cases-1A, 2B and 3B is illustrated in Figure 9. As shown in Figure 9, Reynolds number is independent of the measured depth of horizontal wells. However, Reynolds number is seen to increase with increase in oil withdrawal rate or increase in pressure drop for Cases-1A, 2B and 3B. As expected, similar trend can be observed for the plot of TVD against Re. Figure 9 shows that the Reynolds number increases with an increase in the true vertical depth of the horizontal wells.

$$
R e=\frac{\rho_{o} D_{i} Q_{o}}{\mu_{o} A}
$$

Where $Q_{o}$ is the oil flow rate in cubic meter per second, $D_{i}$ is the inside diameter of the pipe in meters, $\mu_{o}$ is the viscosity of the oil in Newton-second per square meter, $\rho_{o}$ is the density of the oil in Kilogram per cubic meters, $A$ is the cross-sectional area of the pipe in square meters and $R e$ is the Reynolds number in dimensionless unit.

Table 4: Effect of oil withdrawal rates and measured depth on Reynolds number at 300 seconds (exp. ${ }^{-2}$ ).

Cases $\quad$ TVD (m) $\quad$ Measured depth (m) Reynolds number (dimensionless)

\begin{tabular}{lllccc} 
& & & & & \\
\cline { 4 - 6 } Case-1A & 21.70 & 71.80 & 351 Psi & $-7.25 P s i$ & -10.15 Psi \\
Case-1B & 20.30 & 70.10 & 36.11 & 39.24 & 40.32 \\
Case-1C & 19.40 & 69.00 & 36.20 & & \\
Case-2A & 19.00 & 68.30 & 35.33 & & \\
Case-2B & 18.70 & 67.60 & 36.86 & 37.24 & 38.86 \\
Case-2C & 17.40 & 66.40 & 37.78 & & \\
Case-3A & 17.20 & 64.80 & 35.79 & & \\
Case-3B & 16.90 & 64.00 & 37.33 & 37.79 & 39.79 \\
Case-3C & 16.70 & 63.40 & 36.66 & & \\
\hline
\end{tabular}




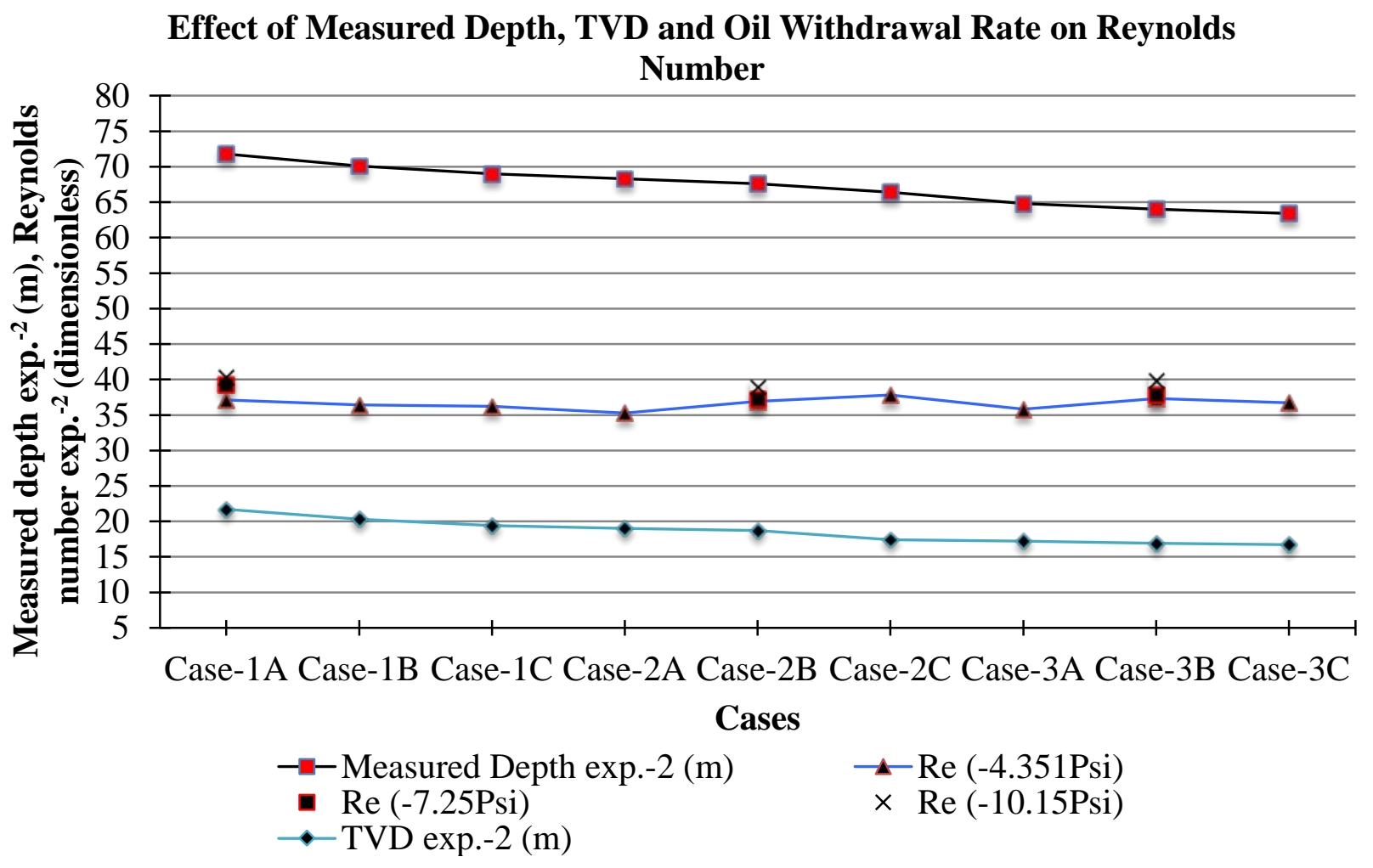

Figure 9: Effect of measured depth, TVD and oil withdrawal rate on Reynolds number at 300 seconds.

\section{Results}

The first step in this investigation was to observe water and gas cresting occurring simultaneously. Figure 10(a) shows the reservoir at static condition prior to oil production, while Figure 10(b) shows the simulation after 60 seconds for Case-1A. In Figure 10(a), the red and black dotted lines represent the initial Water-Oil-Contact and Gas-Oil-Contact respectively whereas the blue and black dotted lines represents the simulated Gas-OilContact and Water-Oil-Contact respectively in Figure 10(b). 


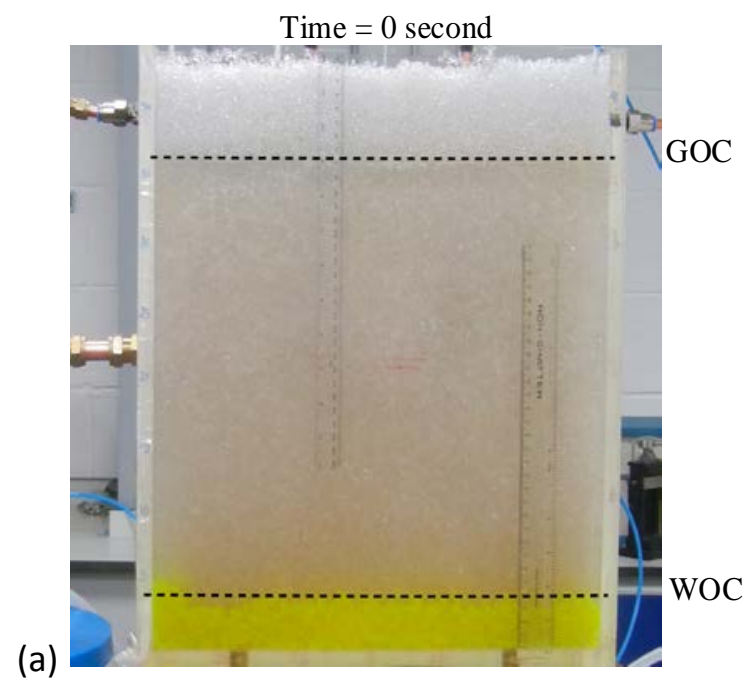

(b)

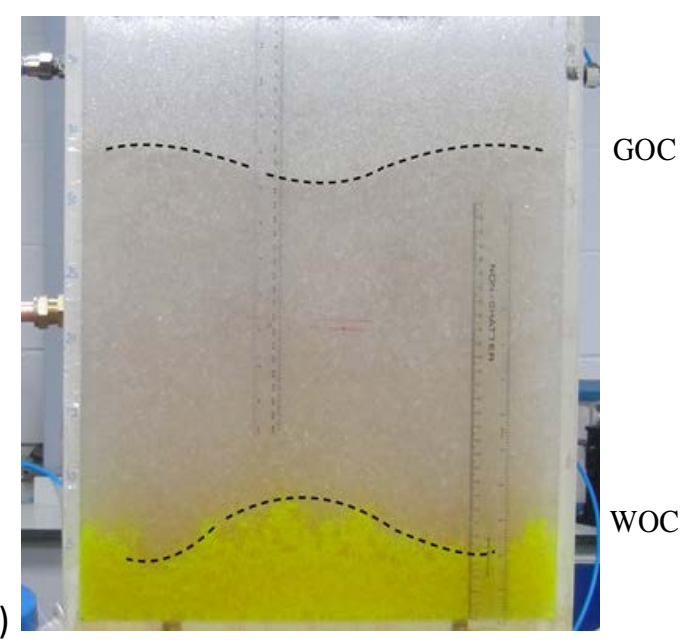

Figure 10: (a) reservoir at static condition (b) Water and Gas cresting occurring simultaneously.

\subsection{Effect on cumulative liquid production rate}

Table 5 illustrates the summary of the cumulative liquid production rates in cubic centimeters per seconds $\left(\mathrm{cm}^{3} / \mathrm{s}\right)$ at a total production time of 300 seconds for all cases while Figures 1113 show a comparison of the cumulative liquid production rates for long radii wells, medium radii and short radii wells respectively. As shown in Figure 11, Case-1A resulted in higher liquid production rate and cumulative barrel of oil produced per second. Oil was produced at a highest cumulative rate of $8.91 \mathrm{~cm}^{3} / \mathrm{s}$, while water was produced at the lowest cumulative rate at $3.70 \mathrm{~cm}^{3} / \mathrm{s}$. Case-2A had the lowest oil production rate $\left(8.48 \mathrm{~cm}^{3} / \mathrm{s}\right)$ and highest water production rate $\left(4.05 \mathrm{~cm}^{3} / \mathrm{s}\right)$. This is due to a shorter well length when compared with Cases$1 \mathrm{~A}$ and $1 \mathrm{~B}$, which explains the fact that at the same operating condition the shorter the well length, the faster the water breakthrough time. Hence, Case-1B succeeded Case-2A with a cumulative water production rate of $3.76 \mathrm{~cm}^{3} / \mathrm{s}$.

Figure 12, follows similar trend for cumulative water production rate depicted in Figure 11. In Figure 12, Case-3A being the shortest in measured depth is seen to have the highest cumulative water production at a rate of $4.27 \mathrm{~cm}^{3} / \mathrm{s}$ and lowest oil production rate at $8.59 \mathrm{~cm}^{3} / \mathrm{s}$. Unlike Case-2A, Case 3A with a measured depth of $6.48 \mathrm{exp} .{ }^{-1} \mathrm{~m}$ was most significant in terms of cumulative liquid production rate for medium radii wells. However, Case-2B is seen to have higher cumulative oil production rate of $8.85 \mathrm{~cm}^{3} / \mathrm{s}$ with considerable cumulative water production rate of $3.83 \mathrm{~cm}^{3} / \mathrm{s}$.

In Figure 13, the cumulative liquid production rates for Cases-2C, 3B and $3 \mathrm{C}$ have higher overall cumulative liquid production rate when compared to medium and long radii wells. This is expected, due to the shorter lengths of wells having slightly lower pressure drop compared to longer lengths of wells. Figure 13 shows that Case-2C produced oil at highest cumulative rate of $9.07 \mathrm{~cm}^{3} / \mathrm{s}$. Case-2C also produced water at the lowest rate $\left(3.41 \mathrm{~cm}^{3} / \mathrm{s}\right)$. More so, Case-2C a short radius horizontal well is seen to have performed best when 
compared to both the long and medium radii horizontal wells, which contradicts Freeborn et al.'s results. However, the result from this study clarifies Freeborn et al.'s reason for poor performance of short radii wells. As earlier stated, the reason for the poor performance of the short radius well was due to completion mechanism and perforation jet issues. However, in this case the possible result of the poor performance of the long and medium radii wells could be due to longer arc lengths (longer horizontal and vertical displacements of the inclined region), resulting in a slightly higher-pressure drop, as well as the more time for the mobility of the fluid(s) to be affected by gravitational pull. A closer observation has been given to the results obtained from Freeborn et al., regarding the performances of the long and medium radii wells with the same completion mechanism. They reported that the long radius well performed better at higher production rates than the medium radius well. This is in contrast with the results presented here. From the results, it was observed that the shorter the length of the well, the higher the liquid production rate, hence the medium radius wells performed better having higher average oil recovery ratios compared to long radius wells. This is because of the uniform pressure distribution along the entire length of the longer radius wells resulting in lower pressure drop and hence lower production rates.

The performance of horizontal wells depends on its length and as such a percentage difference of $6.51 \%$ was observed for cumulative oil production rate between Case-2C and Case-2A, whereas significant percentage difference of $20.14 \%$ was observed between Case2C and Case-3A for cumulative water production rate. In Figures 11 to 13, the water and oil cumulative production rates were very close in all cases, due to very close difference in pressure drop resulting from slight differences in measured depths between the horizontal wells. However, a longer production time, greater than 300 seconds could yield significant difference between all cases.

Table 5: Cumulative liquid production rate at 300 seconds.

\begin{tabular}{cccc} 
Cases & $\begin{array}{c}\text { Cumulative oil } \\
\left(\mathrm{cm}^{3} / \mathrm{s}\right)\end{array}$ & $\begin{array}{c}\text { Cumulative water } \\
\left(\mathrm{cm}^{3} / \mathrm{s}\right)\end{array}$ & $\begin{array}{c}\text { Total cumulative liquid } \\
\left(\mathrm{cm}^{3} / \mathrm{s}\right)\end{array}$ \\
\hline Case-1A & 8.91 & 3.70 & 12.61 \\
\hline Case-1B & 8.74 & 3.76 & 12.50 \\
Case-1C & 8.69 & 3.56 & 12.25 \\
Case-2A & 8.48 & 4.05 & 12.53 \\
Case-2B & 8.85 & 3.83 & 12.68 \\
\hline Case-2C & 9.07 & 3.41 & 12.48 \\
Case-3A & 8.59 & 4.27 & 12.86 \\
\hline Case-3B & 8.96 & 3.56 & 12.52 \\
Case-3C & 8.80 & 3.91 & 12.71 \\
\hline
\end{tabular}




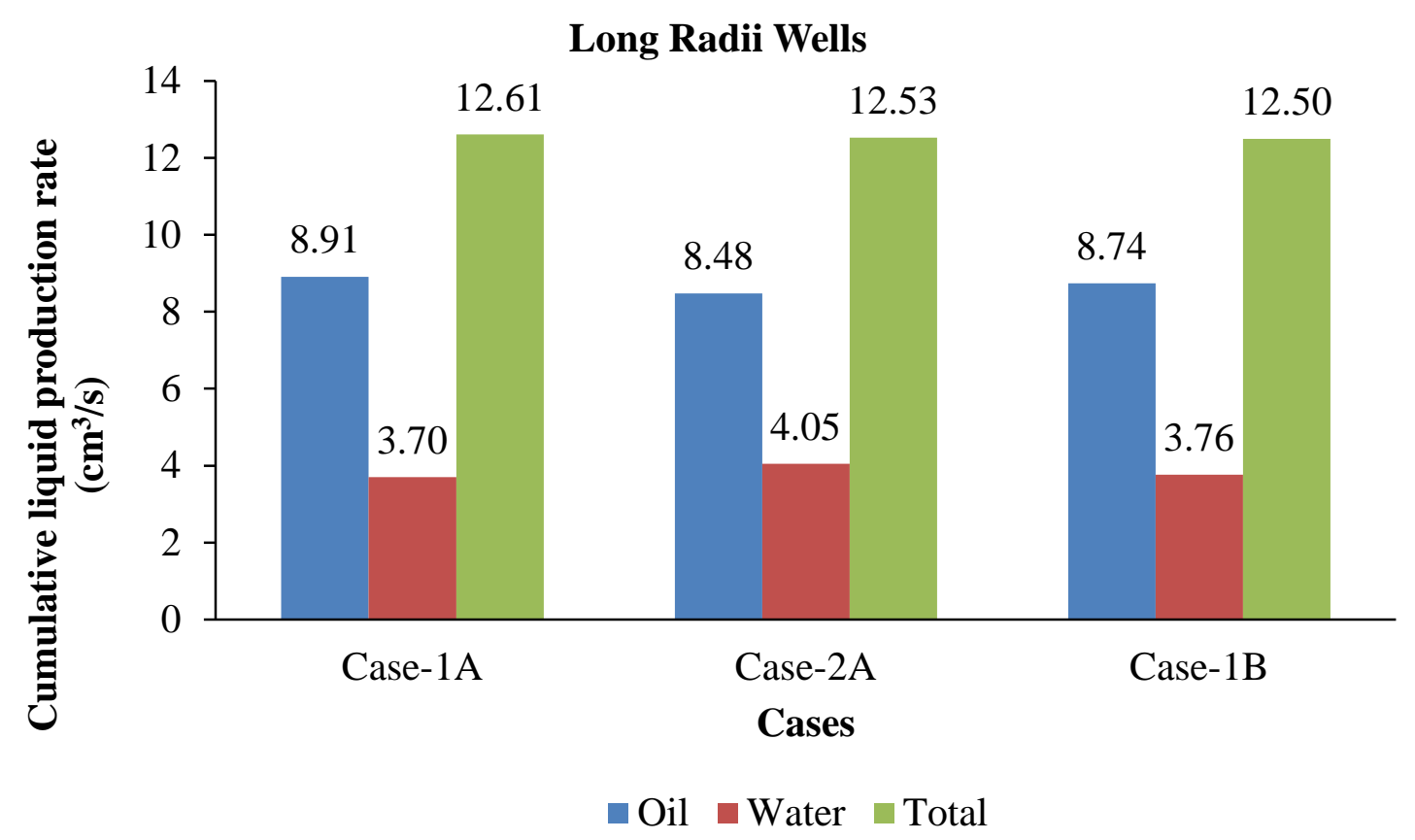

Figure 11: Cumulative liquid production rate comparison for long radii wells at 300 seconds.

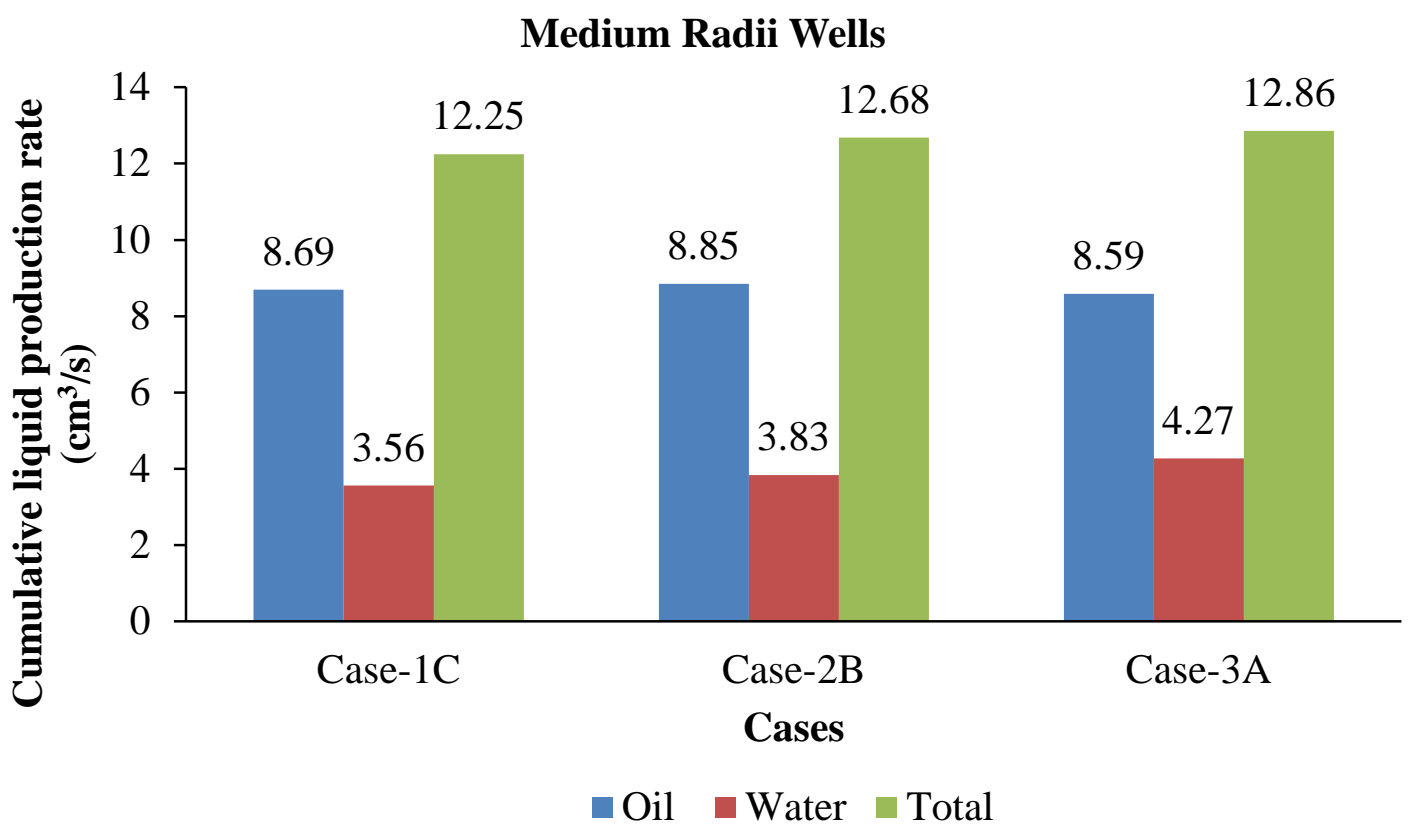

Figure 12: Cumulative liquid production rate comparison for medium radii wells at 300 seconds. 


\section{Short Radii Wells}

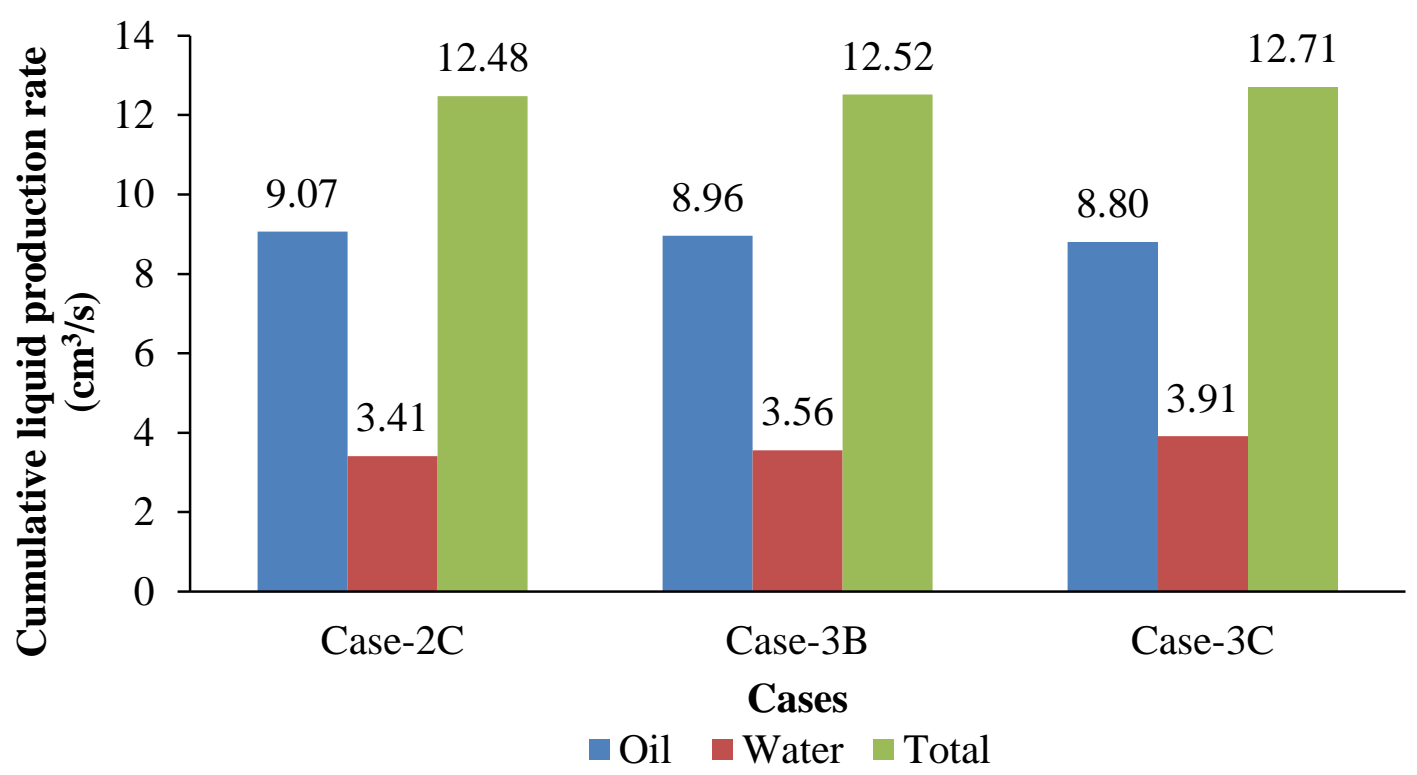

Figure 13: Cumulative liquid production rate comparison for short radii wells at 300 seconds.

\subsection{Effect of increase in pressure drop on cumulative liquid withdrawal rate}

Table 6 is a summary of the effect of increase in pressure drawdown on the cumulative liquid withdrawal rate at 300 seconds. Figures 14-16 show the graphical illustration of the pressure drops increment for Case-1A, case-2B and Case-3B respectively. In Figure 14, an increasing in pressure drop resulted in increasing in both water and oil production rates. For an overall increase in pressure drop of -5.80Psig, an increase of $7.95 \%$ and $1.86 \%$ in oil and water cumulative production rates respectively. As expected, Figures 15 and 16 produced at higher oil and water cumulative production rates; a trend similar to that in Figure 14. Hence for Case-2B an increase of $5.15 \%$ (cumulative oil production rate) and $10.30 \%$ (water cumulative production rate) was observed while there was an increase of $6.18 \%$ and $9.21 \%$ in oil and water cumulative production rates respectively for Case-3B.

The results of the sensitivity analysis performed demonstrate that an increase in pressure drop results in an initial increase in oil produced but accompanied with higher volume of unwanted water. In other words, at higher withdrawal rates, higher cumulative Water-Oil-Ratio over the same production time will be obtained. From the analysis, the longer radius well (Case1A) performed best at a cumulative oil production rate of $9.68 \mathrm{~cm}^{3} / \mathrm{s}$ and least cumulative water production rate at $3.70 \mathrm{~cm}^{3} / \mathrm{s}$. The reason is longer wells have higher pressure drop along its length when compared to shorter wells at the same operating condition, which delays cresting effect and hence highly recommended for economic reasons where high production rates are necessary. 
Table 6: Effect of pressure drop on cumulative liquid withdrawal rate at 300 seconds.

Pressure at surface Cumulative liquid production rate

\begin{tabular}{lccc}
\cline { 2 - 4 } Cases & $(-)(\mathrm{Psig})$ & Oil $\left(\mathrm{cm}^{3} / \mathrm{s}\right)$ & Water $\left(\mathrm{cm}^{3} / \mathrm{s}\right)$ \\
& 4.351 & 8.91 & 3.70 \\
Case-1A & 7.25 & 9.42 & 3.76 \\
& 10.15 & 9.68 & 3.77 \\
& 4.351 & 8.85 & 3.83 \\
Case-2B & 7.25 & 8.94 & 4.20 \\
& 10.15 & 9.33 & 4.27 \\
& 4.351 & 8.96 & 3.55 \\
Case-3B & 7.25 & 9.07 & 3.80 \\
& 10.15 & 9.55 & 3.91 \\
\hline
\end{tabular}

\section{Case-1A}

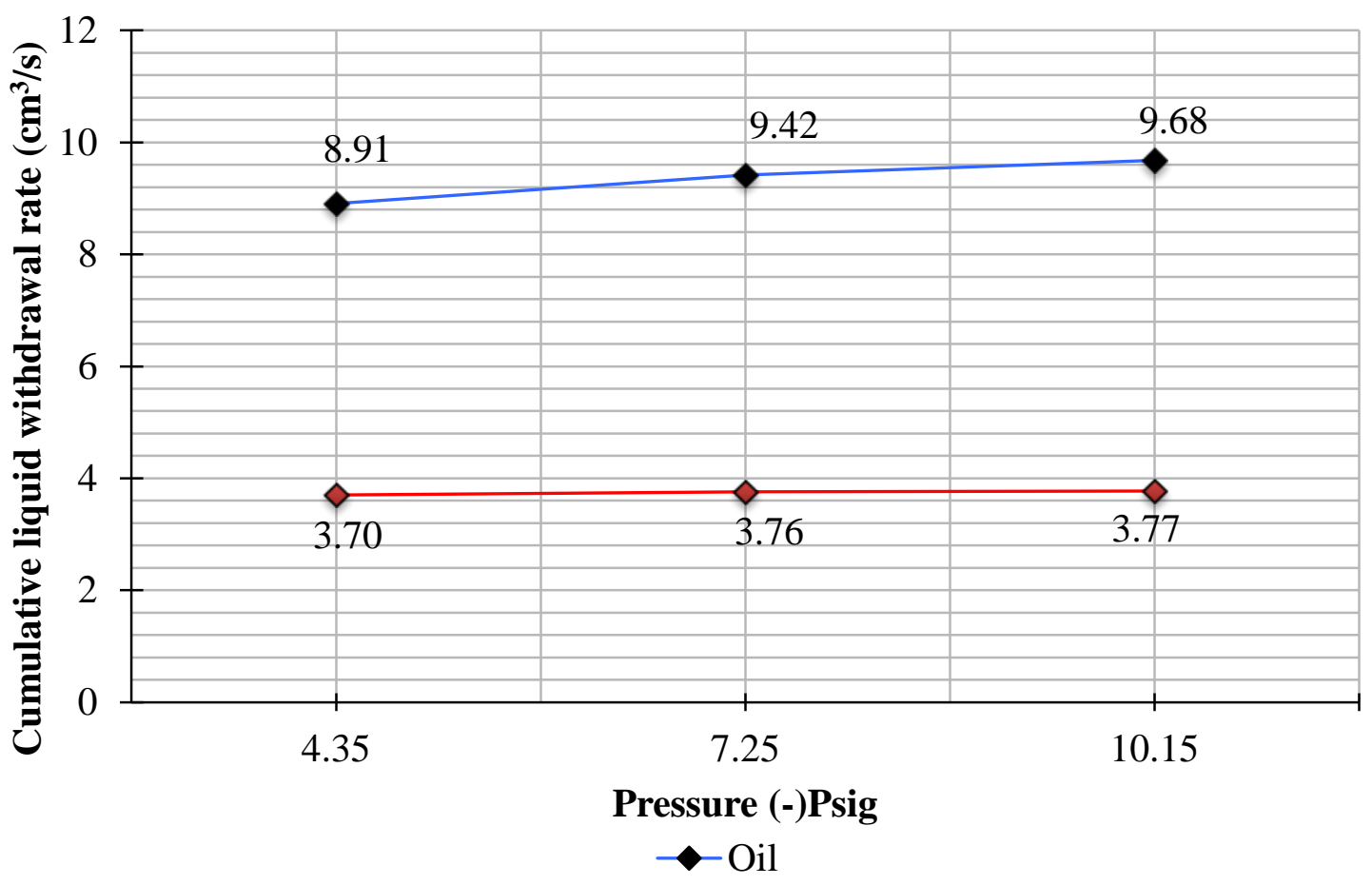

Figure 14: Effect of increase in pressure drop on cumulative liquid withdrawal rate (Case$1 \mathrm{~A})$ at 300 seconds. 


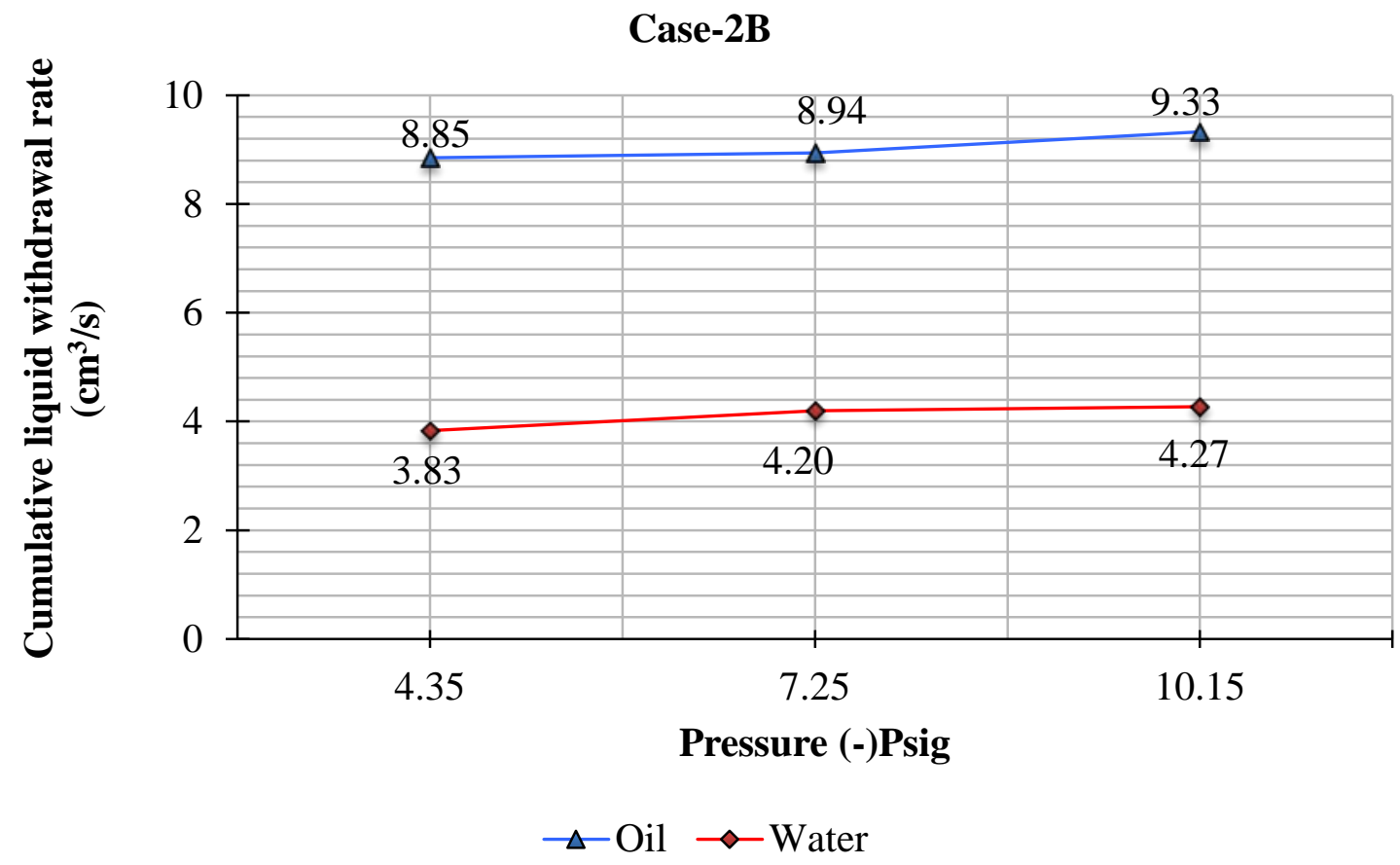

Figure 15: Effect of increase in pressure drop on cumulative liquid withdrawal rate (Case2B) at 300 seconds.

\section{Case-3B}

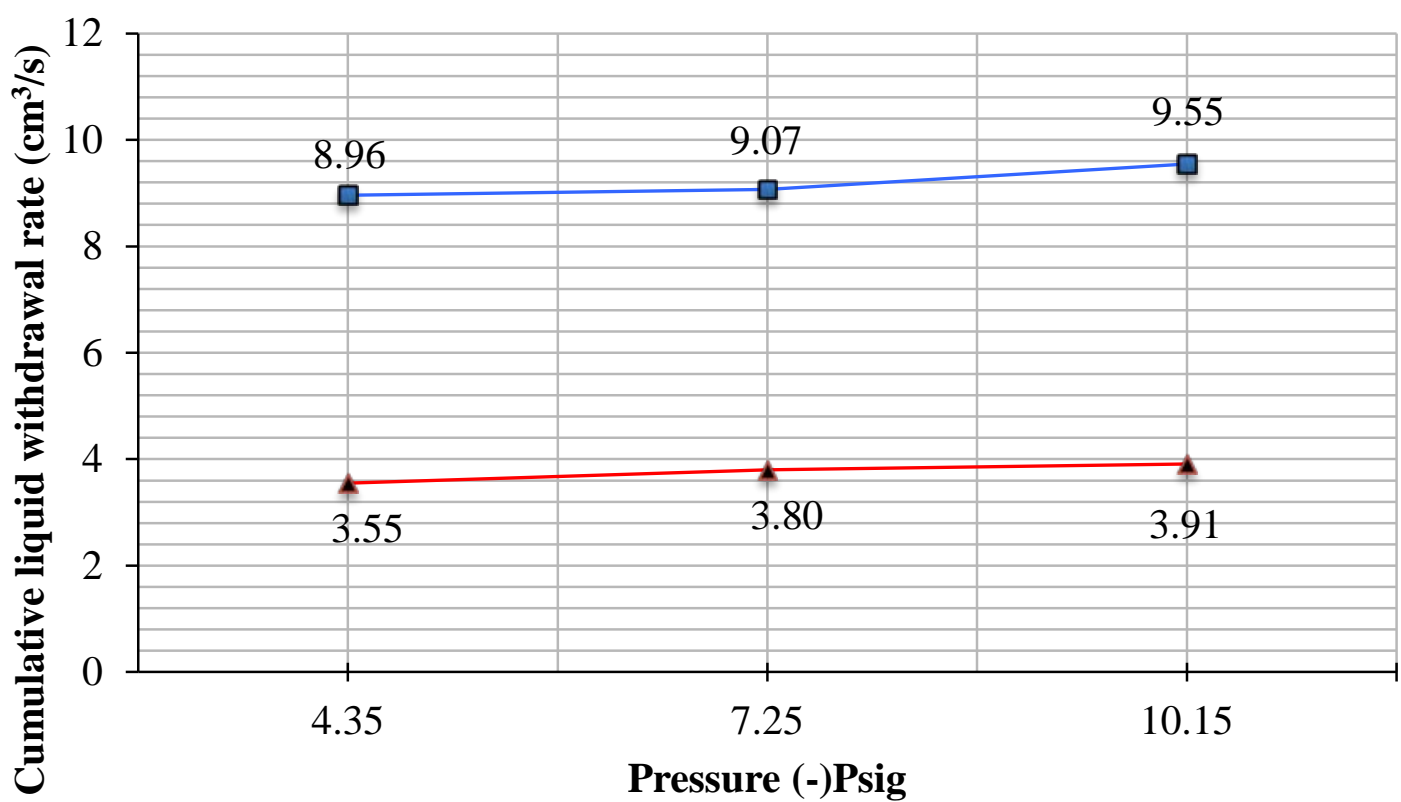

$\rightarrow-$ Oil $\rightarrow$-Water

Figure 16: Effect of increase in pressure drop on cumulative liquid withdrawal rate (Case3B) at 300 seconds. 


\subsection{Effect of varying inclined sections on oil recovery}

Table 7 shows a summary of oil recovery ratio in percentage for the different cases used in this investigation. Figure 17 is a histogram illustrating the data contained in Table 7 for a production time of 300 seconds. From the plot, it can be clearly seen that Case-2C, a medium radius well at $30^{\circ}$ angle of inclination and 0.73 exp. ${ }^{-1} \mathrm{~m}$ horizontal displacement of the inclined section resulted in the highest oil recovery ratio of $38.73 \%$, preceded by Case-3B (0.44m horizontal displacement of the inclined section and $0.70 \mathrm{~m}$ short arc radius) having an oil recovery of $38.26 \%$, while the lowest oil recovery ratio of $36.20 \%$ was obtained from Case-2A (long radius 2.68exp. ${ }^{-1} \mathrm{~m}$ and horizontal displacement of the inclined section, $0.80 \mathrm{~m}$ ). However, the short radii well (Cases-2C, 3C and 3B) had the highest average oil recovery of $38.18 \%$ whereas long radii and medium radii wells had $37.18 \%$ and $37.19 \%$ respectively. Therefore, for a production time of 300 seconds, drilling shorter radii horizontal wells characterized by a lower measured depth and higher fluid mobility (due to lower pressure drop) is recommended for water cresting problems, as this could also be economical in the overall length of pipe used during drilling. However, this may not be the case for longer production times. Interestingly, a percentage difference in oil recovered between the best case (Case-2C) and the case with lowest performance (Case-2A) was 6.53\%. However, for longer production times, larger difference in oil recovered may be obtained.

Table 7: Oil recovery at 300 seconds.

\begin{tabular}{cccc} 
Cases & Original oil in place $\left(\mathrm{cm}^{3}\right)$ & Oil $\left(\mathrm{cm}^{3}\right)$ & Oil recovery (\%) \\
\hline Case-1A & 7027.24 & 2671.78 & 38.02 \\
Case-1B & 7027.24 & 2622.02 & 37.31 \\
Case-1C & 7027.24 & 2605.80 & 37.08 \\
Case-2A & 7027.24 & 2543.80 & 36.20 \\
Case-2B & 7027.24 & 2655.25 & 37.79 \\
Case-2C & 7027.24 & 2721.55 & 38.73 \\
Case-3A & 7027.24 & 2577.98 & 36.69 \\
Case-3B & 7027.24 & 2688.32 & 38.26 \\
Case-3C & 7027.24 & 2638.87 & 37.55 \\
\hline
\end{tabular}


Oil Recovery

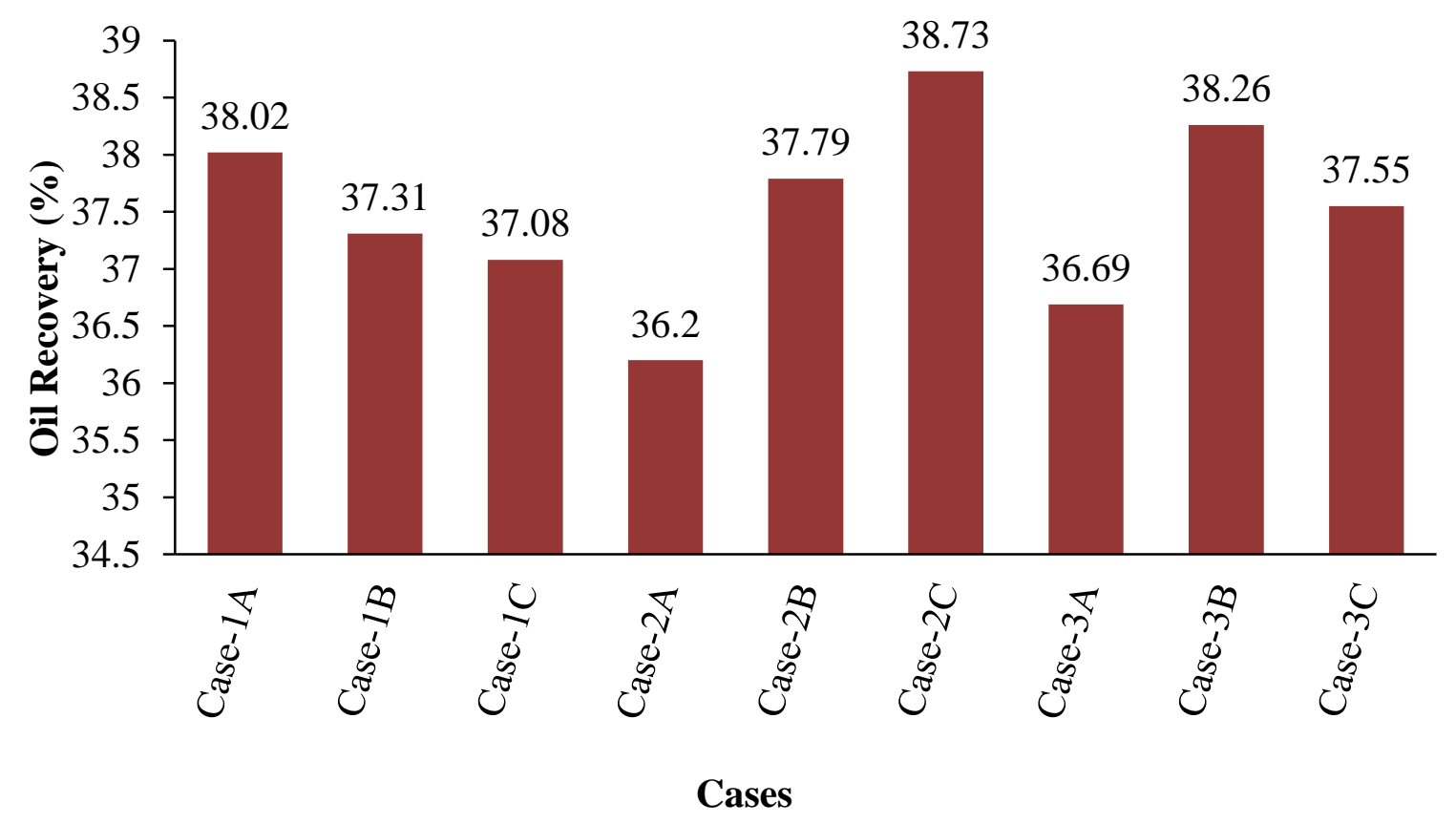

Figure 17: Oil recovery for different horizontal well geometries at 300 seconds.

From the results presented in sections 3.1, 3.2 and 3.3, it is evident that there are differences in the cumulative oil, water production rates and oil recovery for all cases. This is due to differences in measured depths as a result of variations in horizontal and vertical displacements of the inclined section between all horizontal well cases. Hence at the same operating pressure, variation in pressure drop along the lengths of all horizontal wells is expected. Higher cumulative liquid production rate is highest for shorter radii wells (Cases2C, 3C and 3B) due to shorter well lengths when compared to medium (Cases-1C, 2B and $3 \mathrm{~A}$ ) and longer radii (Cases-1A, 2A and 1B) wells. The lower pressure drop values along its length at some point in time during production is also a contributing factor. The same principle is expected for medium and long radii wells but at increasing pressure drop values along their lengths, highest in the later cases.

\section{Conclusions and future work}

In this work a novel experimental procedure was presented for investigating the performance of horizontal wells with varying horizontal and vertical displacements of the inclined section. From the results presented it can be concluded that:

1. The steepness of the inclined section is important in optimizing the productivity of horizontal wells in oil reservoirs affected by severe water cresting. The lower the ratio of the vertical displacement of the inclined section to the reservoir height, the better the performance of the horizontal well in terms of lower Water-Oil-Ratios. 
2. The higher the pressure drop, the higher the water cut due to a higher mass withdrawal rate at any given point in time. In other words, the fluid mobility is dependent on the pressure drop. At low production rates above the critical rate, shorter radii wells are recommended but at higher production rates for economic purposes, the longer radii wells are recommended due to high cresting delay tendency and hence improve oil recovery.

3. Giving reference to the reservoir model and properties described in this paper, good results in terms of higher oil recovery efficiency and least water production rate can be achieved with a vertical displacement of 0.34 exp. ${ }^{-1} \mathrm{~m}$ and 0.07 , with regard to the ratio of vertical displacement of the inclined section to reservoir height.

4. The performance at the inclined section of a horizontal well depends on the angle of inclination and its vertical displacements. For a given geometry, the higher the angle of inclination, the lower the vertical displacement of the build section. Performance of horizontal wells depends on its measured depth.

5. The permeabilities for water, oil and gas were estimated and presented in this study using Darcy's linear flow equation. However, the behavior of flow for water and oil in each case was not presented. This behavior will be investigated in future works by determining the relative permeabilities of the phases involved using an appropriate set up.

6. Using this novel procedure a percentage increment of $5.60 \%$ in oil was recovered with $11.40 \%$ reduction in cumulative produced water from the same reservoir.

\section{References}

Al zarafi, A., 1993. Breathing New Life Into a Thin Oil Column by Horizontal Drilling. Society of Petroleum Engineers. SPE-25532-MS. doi:10.2118/25532-MS.

Balazs, M., Wittmann, G., Vad, J., and Szabo, G., 2009. Experimental modeling of Water and Gas Coning in Horizontal Oil Producing Wells http://www.ara.bme.hu/ szabog/publ/full_text/microCAD_2009_207_leadva.pdf accessed May 2, 2016.

Benamara, A. and Tiab, D., 2001. Gas Coning in Vertical and Horizontal wells, a Numerical Approach. SPE Rocky Mountain Petroleum Technology Conference. Keystone, Colorado. Society of Petroleum Engineers. SPE-71026-MS. doi:10.2118/71026-MS.

Beveridge, S. B., Coats, K. H., and Alexandre, M. T., 1970. Numerical Coning Applications. Petroleum Society of Canada. PETSOC-70-03-07. doi:10.2118/70-03-07.

Chen, H. K., 1993. Performance of Horizontal Wells, Safah Field, Oman. Society of Petroleum Engineers. SPE-25568-MS. doi:10.2118/25568-MS.

Coffin, P., 1993. Horizontal Well Evaluation After 12 Years. Society of Petroleum Engineers. SPE-26618-MS. doi:10.2118/26618-MS. 
Freeborn, W. R., Russell, B. and Macdonald, A. J., 1990. South Jenner Horizontal Wells A Water Coning Case Study. Petroleum Society of Canada. PETSOC-90-03-04. doi:10.2118/90-03-04.

Goode, P. A. and Wilkinson, D. J., 1991. Inflow Performance of Partially Open Horizontal Wells. Society of Petroleum Engineers. SPE-19341-PA. doi: 10.2118/19341-PA.

Kromah, M. J. and Dawe, R. A., 2008. Reduction of Oil and Gas Coning Effects by Production Cycling and Horizontal Wells. Petroleum Science and Technology. 26 (3), 353-367. doi:10.1080/10916460600809501.

Leemhuis, A. P., Belfroid, S. and Alberts, G., 2007. Gas Coning Control for Smart Wells. Society of Petroleum Engineers. SPE-110317-MS. doi: 10.2118/110317-MS.

Lozada, M. A., Poblano, E., Guerrero, I. and Fairuzov, Y. V., 2011. Flow Instabilities in GasLift Wells with Water Coning. Society of Petroleum Engineers. SPE-142694-MS. doi:10.2118/142694-MS.

Makinde, F. A., Adefidipe, O. A. and Craig, A. J., 2011. Water Coning in Horizontal Wells: Prediction of Post-Breakthrough Performance. International Journal of Engineering and Technology. 11 (01).

Murphy, P. J., 1990. Performance of Horizontal Wells in the Helder Field. Society of Petroleum Engineers. SPE-18340-PA. doi:10.2118/18340-PA.

Permadi, P., 1996. Fast Horizontal-Well Coning Evaluation Method. Society of Petroleum Engineers. SPE-37032-MS. doi:10.2118/37032-MS.

Permadi, P. and Jayadi, T., 2010. An Improved Water Coning Calculation for Horizontal Wells. 2010/SPE/RO\&G Russian Oil and Gas Technical Conference held in Moscow, Russia. Society of Petroleum Engineers. SPE-133162-MS. 26-28 October. doi:10.2118/133162-MS.

Sherrard, D. W., Brice, B. W. and Macdonald, D. G., 1987. Application of Horizontal Wells at Prudhoe Bay. Society of Petroleum Engineers. SPE-15376-PA. doi:10.2118/15376-PA.

Singhal, A. K., 1996. Water And Gas Coning/ Cresting A Technology Overview. Petroleum Society of Canada. PETSOC-96-04-06. doi:10.2118/96-04-06. 56-62.

Smith, C. R. and Pirson, S. J., 1963. Water Coning Control in Oil Wells by Fluid Injection. Society of Petroleum Engineers. SPE-613-PA. doi:10.2118/613-PA.

Weijun, S., Xizhe, L., Xiaohua, L. and Jialiang, L., 2014. Investigation of Water Coning Phenomenon in Naturally Fractured Gas Reservoirs. Electronic Journal of Geotechnical Engineering. 18. 1411-1420.

Yang, W. and Wattenbarger, R. A., 1991. Water Coning Calculations for Vertical and Horizontal Wells. Society of Petroleum Engineers. SPE-22931-MS. doi:10.2118/22931-MS. 